Article

\title{
Design and Fabrication of a New Dual-Arm Soft Robotic Manipulator
}

\author{
Yaxi Wang and Qingsong $\mathrm{Xu}$ *D \\ Department of Electromechanical Engineering, Faculty of Science and Technology, University of Macau, \\ Avenida da Universidade, Taipa, Macau, China; mb75425@connect.um.edu.mo \\ * Correspondence: qsxu@umac.mo
}

Received: 28 November 2018; Accepted: 28 December 2018; Published: 4 January 2019

\begin{abstract}
This paper presents the design and implementation of a dual-arm soft robotic manipulator. It consists of two soft manipulators, which are driven by pneumatic actuators. Each soft manipulator is composed of three soft modules, and each module includes three evenly distributed cavities inside. The flexible bending deformation of the soft module is produced by regulating the air pressure and changing the applying sequence to the cavities. The design and fabrication of the manipulator are presented in detail. The cooperation of the dual-arm soft robotic manipulator is implemented by adopting visual servo control. Experimental testing was carried out to demonstrate the manipulator performance. Unlike a single-arm manipulator, the robotic manipulator with dual arms features high flexibility, adaptability, and safety. The feasibility of the proposed dual-arm soft robotic manipulator is demonstrated by executing assembly tasks.
\end{abstract}

Keywords: compliant mechanism; soft robot; soft manipulator; visual servo control; collaborative operation

\section{Introduction}

Currently, 95\% of robots are applied in the industrial field [1]. With the development of modern industries, more and more robots have been adopted for use in other fields, such as surgery, reconnaissance, food picking, etc., increasing the market demand for soft robots. In a previous work, a soft robot was designed for use in minimally invasive surgery [2]. The robot's manipulator is composed of multiple soft modules, which exhibit high flexibility and can be flexibly bent in human intestines. The end of the device is equipped with a surgical tool, which allows for non-invasive examination of the intestine or treatment owing to the high safety of the soft material. A soft robot has abilities that cannot be realized by traditional rigid robots. As compared with regular compliant mechanisms [3-5], soft robots exhibit more advantages in terms of flexibility, adaptability, and safety. Soft robots are typically made of soft or super-elastic material, which is intrinsically soft. Their extendible bodies can deform and absorb energy during operation. Soft robots can be bent, twisted, expanded, and contracted to work in specified workspaces. The satisfactory safety and flexibility of soft robots overcome the defects of rigid robots in various domains, such as minimally invasive surgery and disaster rescue. Moreover, soft robots can better mimic biological prototypes in terms of bionic structures and biomimicry [6].

Soft robots can be driven by different types of actuators. In the literature, many kinds of soft robot actuators have been presented, including the following types: shape memory alloy actuators, pneumatic artificial muscle actuators, fluid elastomer actuators, fiber-reinforced actuators, multifunctional polymer actuators, and so on [6]. These actuators have been adopted in the design of a variety of soft robots, such as a self-contained pneumatic fish, a ribbed planar manipulator, a four-legged soft robot, a jamming soft gripper, a soft manipulator with variable stiffness, etc. 
In comparison with conventional rigid-body robots, soft robots have their own shortcomings. For example, most soft robot actuators currently use fluid control. Due to the nonlinearity of the materials, it is difficult to achieve high precision and high accuracy of control. As a result, work demanding high precision and speed cannot be easily achieved with soft robots, which exhibit much less capacity for these than the conventional rigid robots.

However, conventional rigid joint-link manipulators have limitations in terms of dexterity and kinematic configurations, which make it impossible for some surgical operations to be entirely performed with the same instrument. Although the accuracy of the rigid manipulator is relatively high, its flexibility and ability to adapt to the environment is not satisfactory. Traditional rigid robots are difficult to move freely, for example, in irregular spaces inside the human body. Conventional metal robots perform surgical operations through more complex feedback mechanisms, which may damage human tissue. In contrast, the dexterous structure of soft robots makes it easier for them to change direction or squeeze into narrow spaces.

Generally, the single-arm soft robotic manipulator is designed to perform the same task as that of a rigid manipulator [7-9]. For more dexterous manipulation, a multiple-arm manipulator is required to realize cooperative work. However, there is limited work on soft robots with multiple-arm manipulators in the literature. To this end, in this paper a novel soft robot is designed to realize the cooperation of a dual-arm manipulator. Unlike the soft robots as reported before, the presented robot is implemented with two soft manipulators. In addition, two soft end-effectors with different functions are added to execute dexterous manipulation. The coordinated movement of the two arms can accomplish more complicated work, which can also be used in harsh environments to prevent damage to the human body [10]. In addition, for the dual-arm soft robot, a visual servo control system is implemented based on a binocular camera. The purpose of the control system is to identify the target object and then drive the two soft arms to bend and grasp the target objects for executing the assembly work. The design, fabrication, and testing of the soft robot are presented in detail.

The following parts of the paper are organized as follows. Section 2 presents the mechanism design and fabrication process of the soft modules. The development of the entire soft robotic manipulator is presented in Section 3. Experimental studies with visual servo control and air pressure control design are outlined in Section 4. Section 5 concludes the paper.

\section{Mechanical Design and Fabrication of the Soft Module}

In this work, a dual-arm soft robotic manipulator is designed with two multi-module arms. Each manipulator arm is composed of three soft modules, which are connected in series. Each module (see Figure 1) consists of three chambers, which are driven by three pneumatic actuators, respectively. Thus, the dual-arm manipulator is driven by a total of 18 pneumatic actuators. This section presents the details for the design and fabrication of a single soft module.

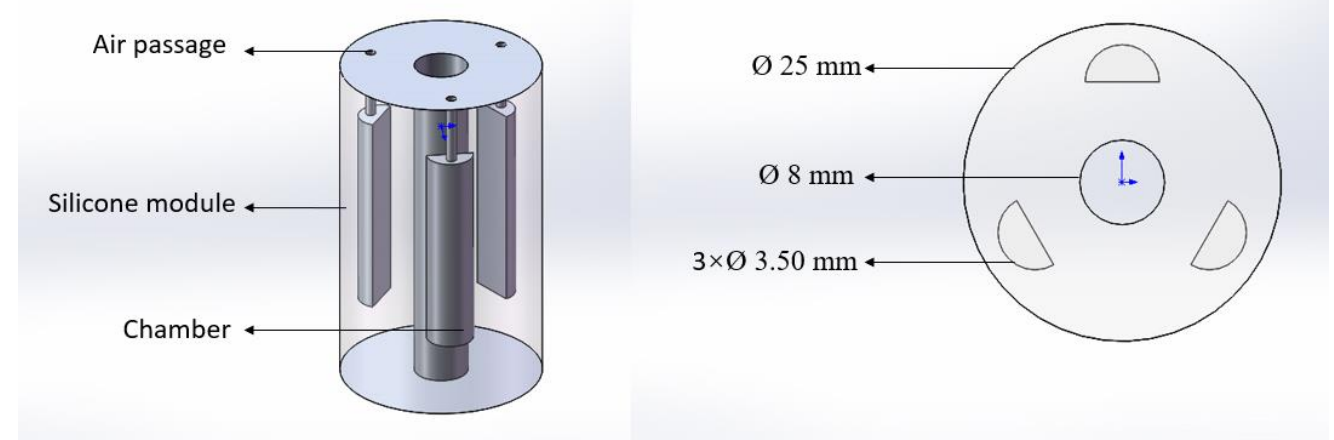

Figure 1. Computer-aided design (CAD) model of a single soft module and the cross-sectional dimensions. 


\subsection{Modeling and Finite-Element Analysis (FEA) Simulation}

Silicone material (e.g., Ecoflex-0030, Smooth-On, Inc., Macungie, PA, USA) is anisotropic, ultra-elastic, and incompressible. It is ideal for making the joints and links of soft robots [11]. To analyze the soft robot properties, the strain potential energy method is usually adopted. The expression for the strain potential energy of Ecoflex silicon is obtained as:

$$
\mathrm{U}=\sum_{i+j=1}^{N} C_{i j}\left(I_{1}-3\right)^{i}\left(I_{2}-3\right)^{j}+\sum_{i=1}^{N} \frac{1}{D_{i}}\left(J_{e}-1\right)^{2 i}
$$

where $U$ is the potential strain energy per unit volume, $N$ is the polynomial order, $I_{1}$ and $I_{2}$ are the deviatoric strain invariants, $J_{e}$ is the elastic volume strain, $C_{i j}$ is the material related parameter for shear behavior, and $D_{i}$ defines the compressibility.

Under the assumption of a fully incompressible material, the models of different order were investigated by fitting the mechanical test data for Ecoflex-0030 [11], where the strain potential energy is irrelevant to the second invariant with $j=0$, that is,

$$
\mathrm{U}=C_{10}\left(I_{1}-3\right)+C_{20}\left(I_{1}-3\right)^{2}+C_{30}\left(I_{1}-3\right)^{3} .
$$

Once the model of the soft module was established, based on the CAD model and ANSYS software, as well as the performance of the material, different pressures of $0,0.05,0.075$, and 0.10 bar were applied to the model of one chamber of the soft module. FEA simulation study of one chamber was performed by applying air pressure to the chamber. The simulation results are shown in Figure 2. It can be seen observed that the bending angle increased as the pressure value increased. Thus, multiple soft modules with flexible bending performance can be used to construct a soft manipulator arm with multiple degrees of freedom.

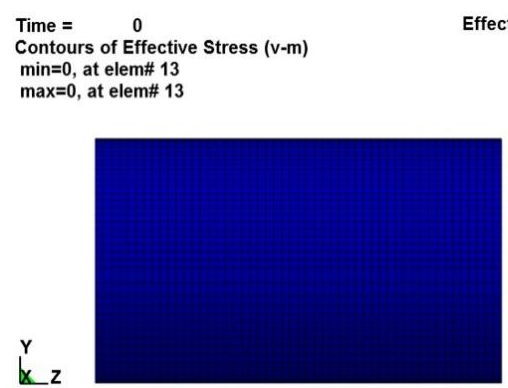

(a)

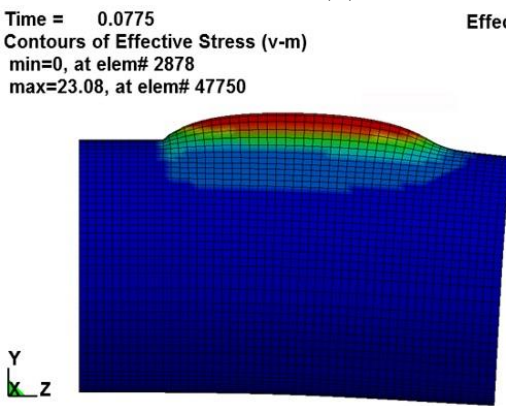

(c)

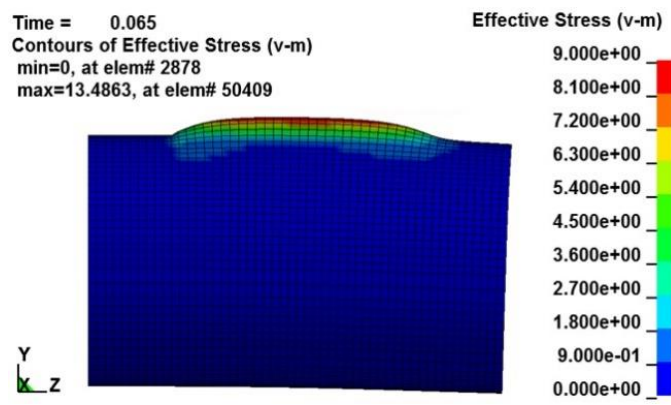

(b)

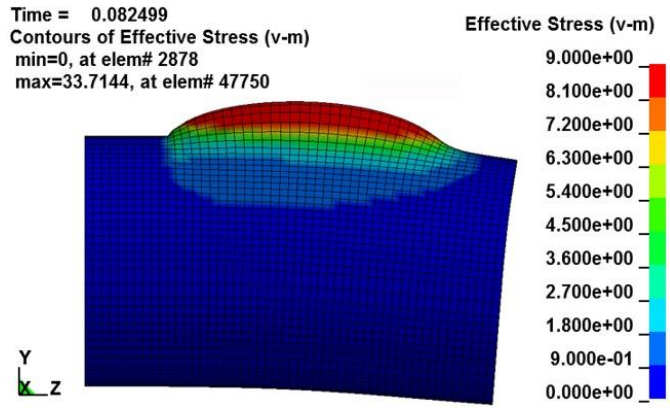

(d)

Figure 2. Finite-element analysis (FEA) simulation results of the soft module by applying a pressure value of (a) 0 bar, (b) 0.05 bar, (c) 0.075 bar, and (d) 0.10 bar.

\subsection{Fabrication of Single Soft Module}

As shown in Figure 3, the fabrication process of a single soft module of the manipulator included the following steps. 
(1) Prepare the Ecoflex-0030 silicone and the 3D-printed molds.

(2) Assemble the mold, close the shells around it, and pour the silicone up to the edge of the shells, and then place the mold in an oven at $60^{\circ} \mathrm{C}$ for about $30 \mathrm{~min}$. Next, remove the shells and cap-A and reverse the module and insert cap-B. Then, close the shells so that their edges are aligned with the stiffening cylinder.

(3) Put the mold into the oven at $60^{\circ} \mathrm{C}$ for about $30 \mathrm{~min}$. Remove the external shells, cap- $\mathrm{B}$, chambers, and the stiffening cylinder.

(4) Insert the actuation tubes and cut three silicone tubes to the same desired length ( $300 \mathrm{~mm}$ in this work).

(5) Put silicone glue around one end of each tube, without obstructing the tubes. Insert three tubes with dedicated air channels into the module.

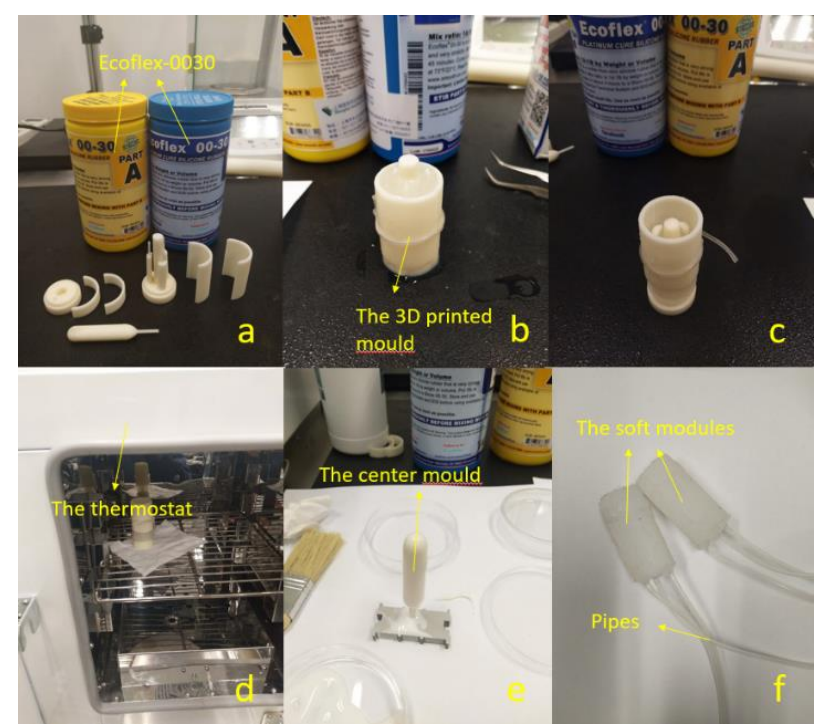

Figure 3. Fabrication process of a single soft module.

According to the above steps, six identical soft modules were fabricated and combined to construct the dual-arm soft robotic manipulator. The manufacturing of the soft module was conducted manually. It was important to strictly control the time and environment (e.g., temperature) of each link fabrication. Otherwise, the produced different modules may have had different bending performance, which is unfavorable for the control of the constructed soft robot. Thus, it was necessary to ensure that the material components of the six modules, the ambient temperature, and the time of fabricating each link were as consistent as possible. In addition, in the process of material combination, it was also necessary to ensure that there are no large bubbles in the liquid. Otherwise, the bending performance of the module would be affected.

\subsection{Testing Results of a Soft Module}

Once the individual modules were fabricated, the relationship between the bending angle of the module and the applied pressure value of the cavities was obtained by experimental test. The test of the bending deformation of each module is necessary, as it is useful for realizing the stereo vision-based visual servo control of the entire robot.

As shown in Figure 4, the experimental test was performed on an individual module. Each module consisted of three evenly distributed air cavities. The bending angle could be varied by applying pneumatic pressure to each chamber and changing the application sequence and air pressure value for the three cavities. Thus, the test was conducted in three steps. 

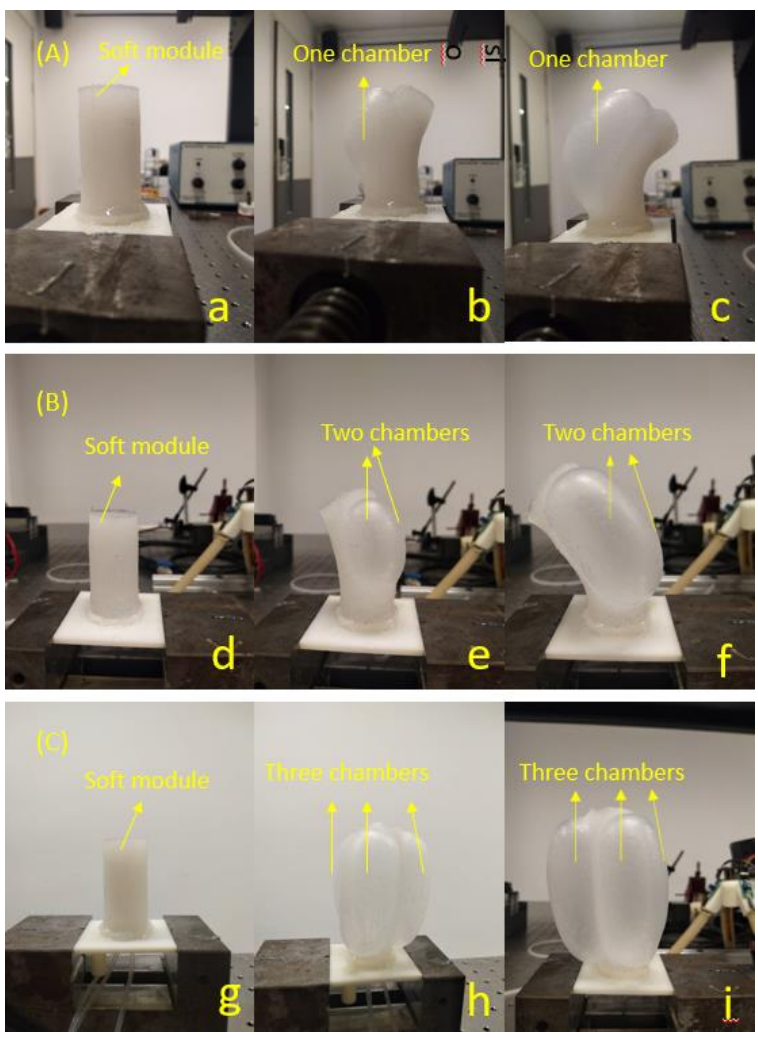

Figure 4. Result of applying air pressure to a single chamber (A), two chambers simultaneously (B), and three chambers simultaneously $(\mathbf{C})$.

First, the air pressure was applied to a single chamber of the soft module. By changing the air pressure value, the bending angle of the module was varied as shown in Figure 4A. The relationships between the bending angle and air pressure of the three chambers are shown in Figure 5a, where the results of three testing times exhibited a consistent relationship.

Second, the air pressure was applied to two chambers simultaneously. By changing the air pressure value, the bending angle of the modules was varied as shown in Figure $4 \mathrm{~B}$. The bending direction of the two modules changed by $120^{\circ}$ compared with that in the first step. In addition, when the air pressure was applied to the two cavities with different values, the bending direction of the modules varied from 0 to $120^{\circ}$. This performance is important for the soft manipulator to achieve flexible manipulation. The relationships between the bending angle and air pressure of the three groups of two chambers are shown in Figure 5b.

From the foregoing two experimental tests, the data of the bending degrees and corresponding pressure values were obtained. Using these data for nonlinear fitting, the relationship between the bending angle and pressure value was generated as below:

$$
\mathrm{W}=85.7 p^{2}+540 p-12.4,
$$

where $\mathrm{W}$ is the bending angle $\left(^{\circ}\right)$ and $p$ is the air pressure (bar).

Third, the air pressure was applied to three chambers simultaneously, as shown in Figure 4C. It was found that the length of the modules varied, demonstrating that the soft module had a certain degree of expansion motion, which also contributed to the flexibility of the soft manipulator. The relationship between the elongation length and the applied air pressure is shown in Figure $5 c$, where the results of three times testing are given. 
Similar to the previous two experiments, nonlinear fitting was adopted to derive the relationship between the elongation length and pressure value as follows:

$$
\mathrm{L}=-657 p^{2}+431+33.2
$$

where $\mathrm{L}$ represents the elongation length $(\mathrm{mm})$ and $p$ denotes the air pressure (bar).
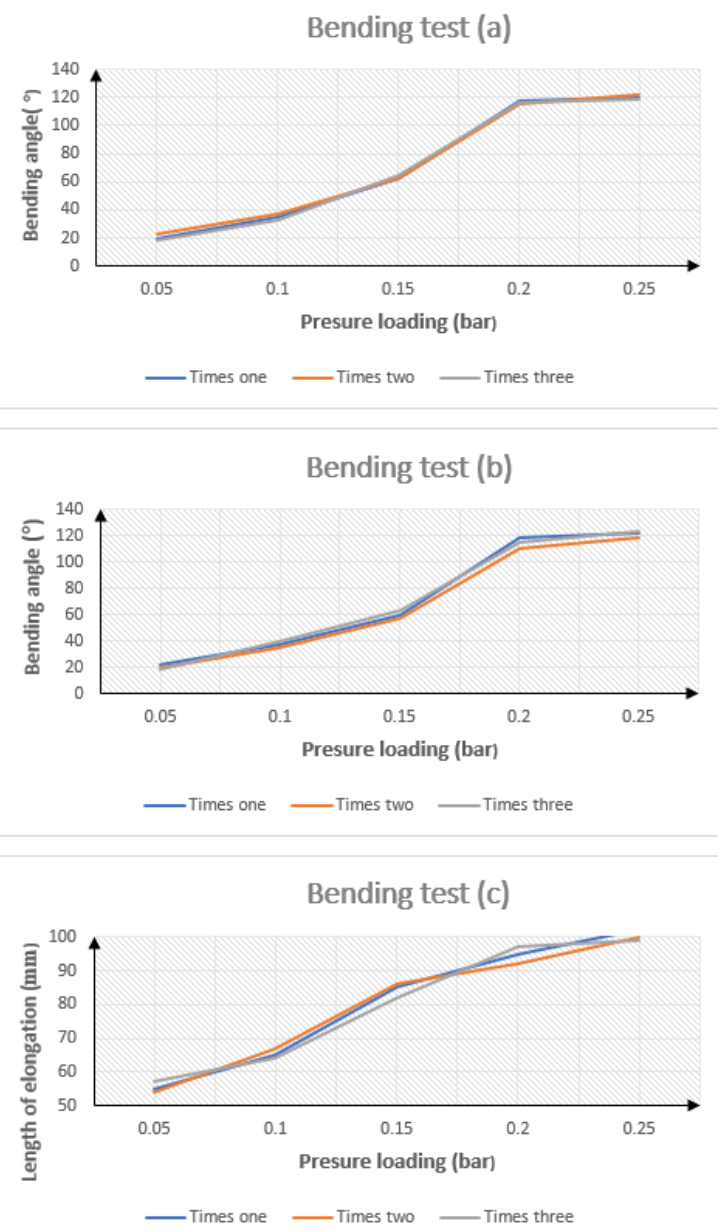

Figure 5. Bending angle of the soft module when one chamber is actuated (a), two chambers are actuated simultaneously (b), and three chambers are actuated simultaneously (c).

It can be seen from Figure 5 that the application of air pressure to one chamber and two simultaneous chambers induced different bending angles of the soft module. The aforementioned experimental results exhibit the relationship between the bending angle and elongation length of the soft module versus the applied air pressure. These relationships are useful for the control of the manipulator to the target position. Since the six modules were made of the same material following the identical fabrication process, there was little difference between their performances.

\section{Prototype Development}

\subsection{Fabrication of Soft Manipulator Arms}

Since the individual modules are short in length, it is not easy to reach a large workspace in space. Thus, it is necessary to integrate multiple soft modules together to construct a manipulator arm. In this work, three soft modules were connected together to construct one manipulator arm.

As shown in Figure 6, an integrated soft arm was fabricated by the following steps. 
(1) Fabricate the granular jamming membrane. The membrane fabrication is based on Latex material because it ensures good performance when the vacuum is applied.

(2) Connect three granular jamming membranes in series.

(3) Cut two plastic tubes to $12 \mathrm{~mm}$ length and one tube to a longer desired length (300 $\mathrm{mm}$ ).

(4) Insert the granular jamming membranes into the manipulator arm [9].

By the foregoing procedure, two identical manipulator arms were fabricated.

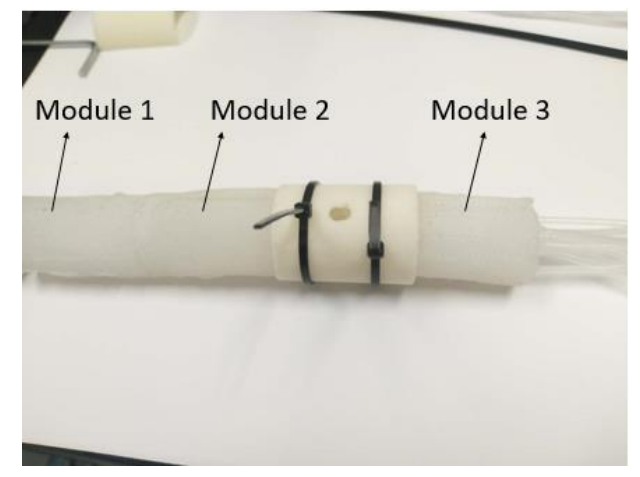

Figure 6. A soft manipulator arm with three soft modules.

\subsection{Fabrication of Soft End-Effectors}

By applying different air pressure values to each cavity and changing the applying order of the air pressure, the end of each soft arm can be positioned to the target position in space. For the execution of manipulation tasks, it is necessary to mount suitable end-effectors at the arm ends.

In this work, two types of soft end-effectors were fabricated as shown in Figure 7. One end-effector was a two-jaw gripper, casted from a black rubber material with a gap of $3 \mathrm{~mm}$ between the two fingers. A silicone tube was connected to the input end of the actuator. Negative input air pressure causes the actuator to open; while positive input air pressure causes the finger to close. Since the actuator is composed of two parallel fingers, it can be used to grasp a longer object, such as a rectangular parallelepiped [12,13]. Another end-effector was a four-jaw gripper, consisting of four evenly circular fingers with a 3-mm gap between each pair of adjacent fingers. A silicone tube was connected to its input end.

The two soft manipulator arms with soft end-effectors are illustrated in Figure 7.

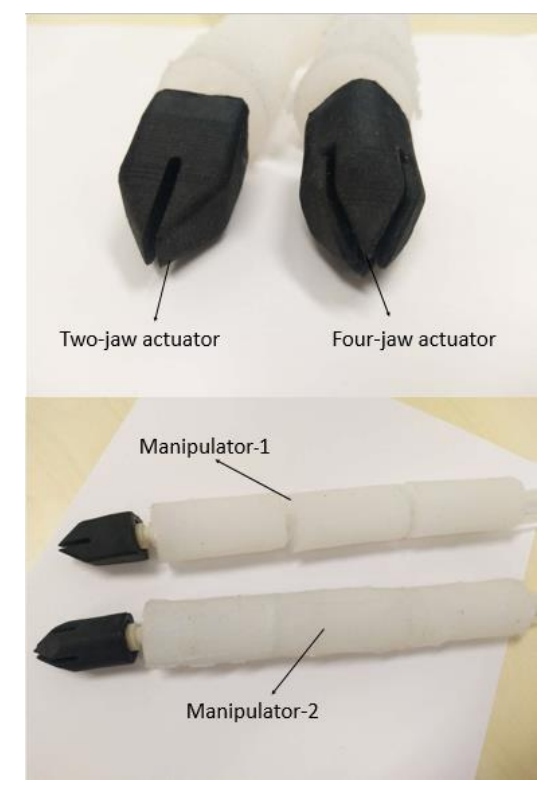

Figure 7. The fabricated soft manipulator arms with soft end-effectors. 


\subsection{Fabrication of a Dual-Arm Soft Robotic Manipulator}

By mounting the two soft arms with a separation distance of $70 \mathrm{~mm}$, the entire dual-arm soft robotic manipulator was constructed as shown in Figure 8. The entire soft robotic manipulator consisted of a total of 20 tubes, which were controlled by closing/opening/changing the air pressures of 20-way gas circuit. This was implemented by using 20 solenoid valves and an air compressor. In addition, a binocular camera was installed for object detection and identification [14,15].

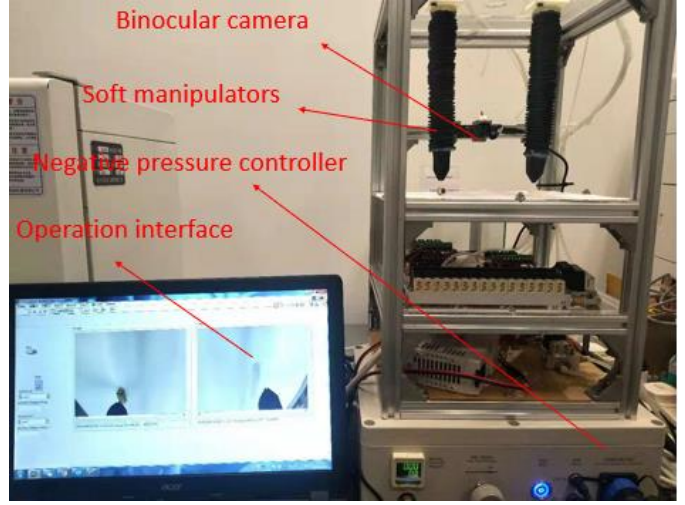

(a)

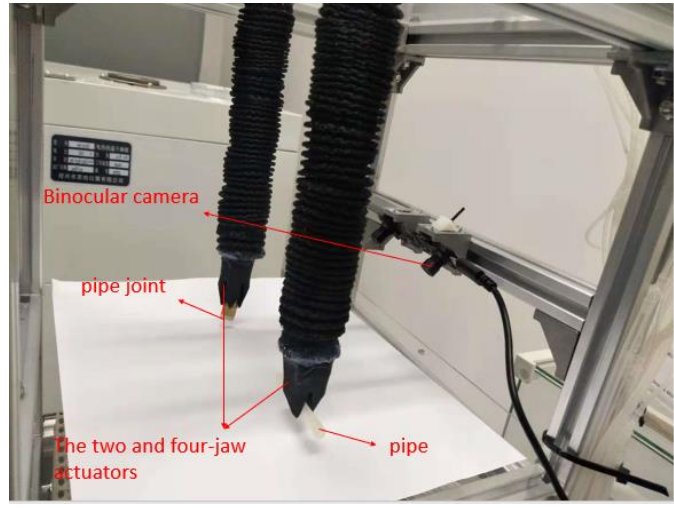

(b)

Figure 8. Prototype and experimental setup. (a) Experimental setup of the dual-arm soft robotic manipulator; (b) close-up view of the experimental setup.

\section{Experimental Studies and Results}

In this work, visual servo control was implemented for the dual-arm soft robotic manipulator to execute the assembly task. Specifically, a binocular camera was adopted to acquire the images. As the two arms work together, it is required to capture the coordinate position of the target object separately, then drive the soft arm to move and grab the target object, and finally realize the assembly work.

\subsection{Design of Visual Servo Control System}

The visual servo control system was composed of both hardware and software. The hardware mainly consisted of an Arduino controller, a solenoid valve, an air source, a negative pressure generator, and a binocular camera. The software was implemented by adopting LabView Vision Assistant Toolkit and LabView plug-in kit for the Arduino development environment. During the control process, the camera is used to recognize the position of the target object. The position coordinate information was fed to the LabView control program, and the control program sent the control signal to the Arduino microcontroller for driving the solenoid valves to open or close. In this way, the bending of the two soft arms, grasping of target object, and assembly tasks were achieved.

The visual servo control system used a USB binocular camera. Since the process of image acquisition and processing involves the conversion between coordinate systems, different coordinate systems were established, namely, the camera coordinate system, world coordinate system, and image coordinate system [16]. The imaging system met the following requirements:

$$
\frac{1}{f}=\frac{1}{u}+\frac{1}{v^{\prime}}
$$

where $f$ is the focal length of the camera, $u$ is the distance between the image and the camera, and $v$ is the distance between the object and lens.

The transformation matrix and translation vector can be used to form a transformation to express the relationship between the camera and world coordinate systems. If the coordinate of the world 
coordinate system is $\left(X_{w}, Y_{w}, Z_{w}, 1\right)^{T}$ and the coordinate of the camera is $\left(X_{c}, Y_{c}, Z_{c}, 1\right)^{T}$, then the conversion is realized as follows:

$$
\left[\begin{array}{c}
X_{c} \\
Y_{c} \\
Z_{c} \\
1
\end{array}\right]=\left[\begin{array}{cc}
R & t \\
0^{T} & 1
\end{array}\right]\left[\begin{array}{c}
X_{w} \\
Y_{w} \\
Z_{w} \\
1
\end{array}\right]=M_{1}\left[\begin{array}{c}
X_{w} \\
Y_{w} \\
Z_{w} \\
1
\end{array}\right]
$$

where $R$ is a $3 \times 3$ matrix, $t$ is the translation vector, and $M_{1}$ is a $4 \times 4$ matrix. In addition, $0^{T}=$ $(0,0,0)^{T}$.

Therefore, the relationship between the camera and image coordinate systems can be established as follows:

$$
Z_{c}\left[\begin{array}{c}
u \\
v \\
1
\end{array}\right]=\left[\begin{array}{ccc}
\frac{1}{d x} & 0 & u_{0} \\
0 & \frac{1}{d y} & v_{0} \\
0 & 0 & 1
\end{array}\right]\left[\begin{array}{cccc}
f & 0 & 0 & 0 \\
0 & f & 0 & 0 \\
0 & 0 & 1 & 0
\end{array}\right]\left[\begin{array}{cc}
R & t \\
0^{T} & 1
\end{array}\right]\left[\begin{array}{c}
X_{w} \\
Y_{w} \\
Z_{w} \\
1
\end{array}\right],
$$

where $(d x, d y)$ represents the scale of a pixel and $\left(u_{0}, v_{0}\right)$ is the origin of the image coordinate in pixels.

In order to achieve the coordinated work of the two manipulators with high motion accuracy, one must effectively control the bending of the manipulator to arrive at the desired position. In the traditional rigid robot field, there is an effective feedback control method called stereo vision feedback control. Stereoscopic vision is one way to obtain the motion and position by acquiring and processing the images $[17,18]$. In fact, the vision servo control system can also be applied in the field of soft robotics. The vision servo control was implemented based on the designed soft robotic manipulator. The manipulator arms were bent to close to the target object, and the end-effectors were used to grasp and assemble the target objects $[19,20]$.

The control block diagram of the entire soft robotic manipulator is shown in Figure 9. The workflow of the visual servo control system is described as follows:

(1) Calibrate the binocular camera.

(2) Capture the images and identify the target objects.

(3) Calculate the pressure value to be driven according to the coordinates of the target objects.

(4) Give the driving signal output using LabView for Arduino toolkit.

(5) Each arm of the two-armed soft robot bends and grabs the target object, respectively.

(6) One target object (i.e., the pipe joint) is assembled with another object (i.e., the air pipe), and then placed back in a desired position.

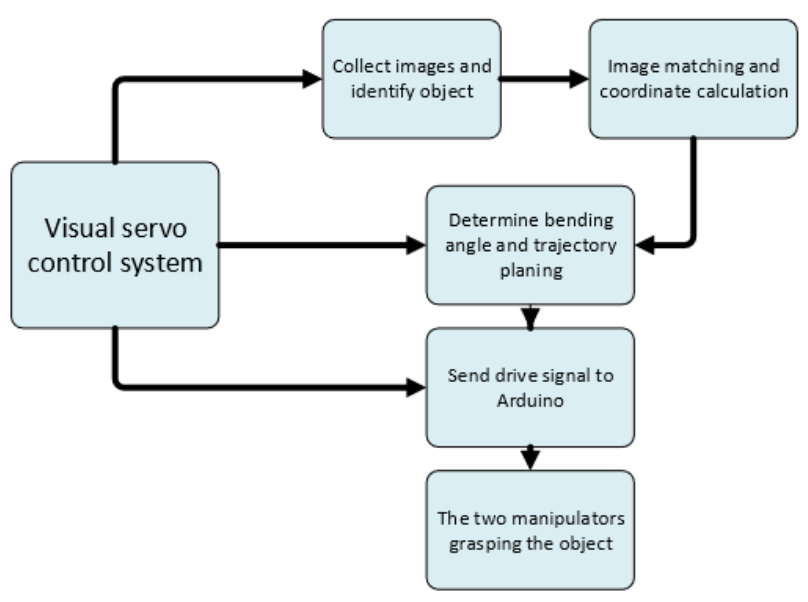

Figure 9. Control block diagram of the two-arm soft robotic manipulator. 
The purpose of camera calibration is to establish the relationship between the coordinates of a point on a spatial surface of an object and its mapping coordinate in the image plane [21]. Thus, the calibration of the binocular camera is a prerequisite for identifying the target object, and is also a necessary factor for calculating the coordinates of the target object. In particular, the calibration of the binocular camera was implemented by using the LabView Vision Assistant Toolkit.

\subsection{Design of Air Pressure Control System}

As shown in Figure 8b, to drive the end-effectors to any position in the working range, an air pressure control system was designed for the two soft manipulators. The control system was powered by a $24 \mathrm{~V}$ direct-current (DC) power source, which was connected to two voltage regulating modules. One was used to power the drive modules, which were used to drive 20 electromagnetic valves (model: VQ110-5M, from SMC Corp., Yorba Linda, CA, USA). The 20 electromagnetic valves controlled the bending degree of the soft robotic manipulator through 20 air pipes. Another regulating module was used to power the electronic regulator, which could automatically adjust the output pressure [22]. Specifically, the analog voltage of $0-10 \mathrm{~V}$ could be set to adjust the pressure value of $0-0.9 \mathrm{MPa}$. A microcontroller (Arduino Mega 2560, Arduino, Somerville, MA, USA) was connected with the electronic pressure regulating valve to realize the communication between them. Real-time control of the air pressure was implemented by the Arduino microcontroller through a connected PC [23,24].

\subsection{Experimental Results of Assembly}

In this work, the dual-arm soft robotic manipulator was used to realize the assembly task of a pipe joint and a pipe under the visual servo control system and air pressure control system. This is important for achieving the desired movement of the soft arms [25,26].

In particular, the control system for each air pressure of soft manipulator was used to drive the two end-effectors of the soft manipulator. By identifying the positions of the target objects, the coordinate positions of the target objects were calculated based on the calibration data. Then, the two arms were driven to approach the target objects. Finally, the target objects were grasped by the end-effectors and assembled together. Snapshots of the experimental process are shown in Figure 10.

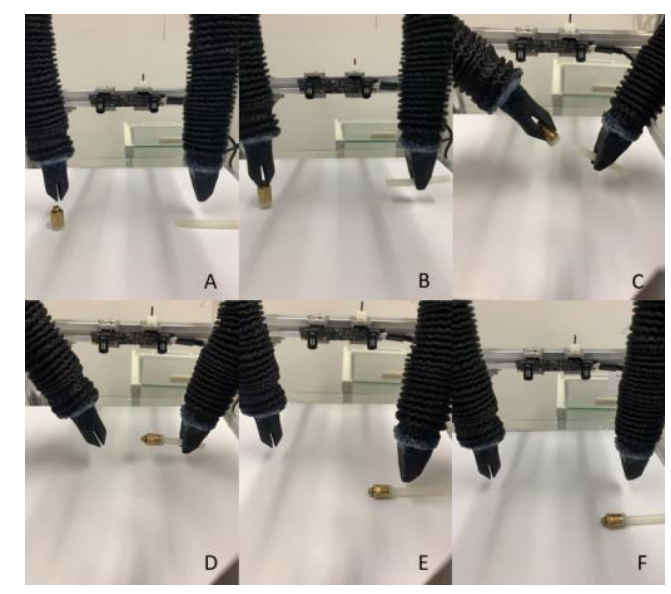

Figure 10. Results of joint and pipe assembly. (A) Identify the location; (B) Approach the object; (C) Start the assembly; (D) Complete the assembly; (E) Place the object in the specified location; (F) Return the arms to their original positions.

The assembly experiment was repeated ten times, and the results are summarized in Table 1. From the experimental results, it can be seen that the developed dual-arm soft robotic manipulator had a success rate of $90 \%$ for this assembly task. The average working time was $12.95 \mathrm{~s}$. Thus, the soft manipulator exhibited a relatively high work efficiency, which demonstrates the effectiveness of the developed soft robot system. 
Table 1. The working time and successful cases of 10 assembly experiments.

\begin{tabular}{ccc}
\hline Serial Number & Succeed or Not & Time (s) \\
\hline 1 & succeed & 12.3 \\
2 & succeed & 13.1 \\
3 & succeed & 13.1 \\
4 & not & 0 \\
5 & succeed & 13.0 \\
6 & succeed & 12.7 \\
7 & succeed & 12.4 \\
8 & succeed & 13.3 \\
9 & succeed & 13.3 \\
10 & succeed & 13.4 \\
\hline
\end{tabular}

Moreover, the final position errors of ten times of assembly experiments are shown in Figure 11. Due to the high flexibility of the soft end-effectors, the position errors of nine successful experiments laid within the range from -4 to $4 \mathrm{~mm}$. Within this error range, the dual-arm soft robot could complete the assembly task successfully in this work. It was observed that the final position error $(5.5 \mathrm{~mm})$ of the fourth experiment exceeded the allowable range, and the assembly task was unsuccessful.

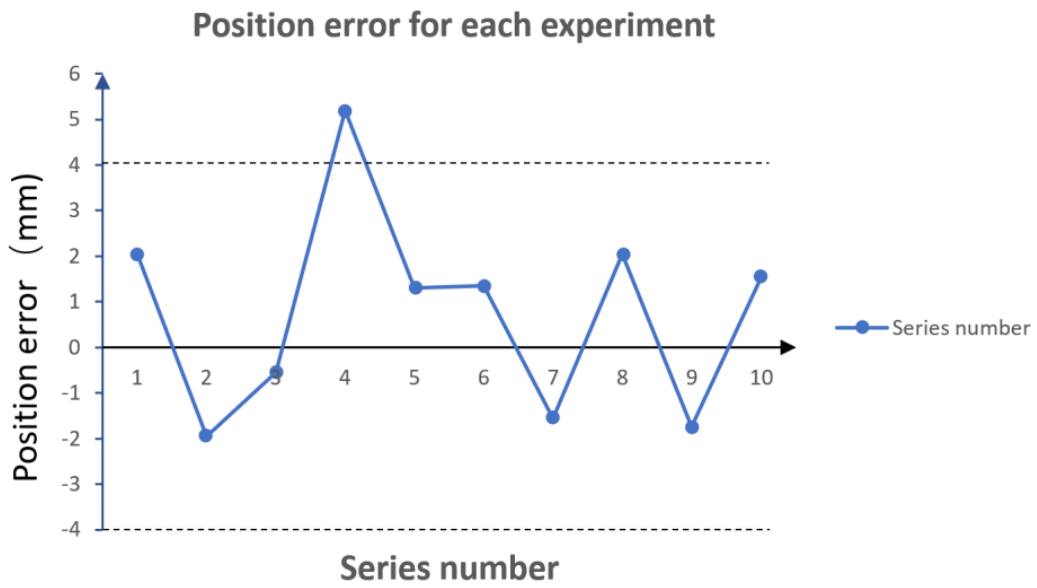

Figure 11. Position errors of ten repetitions of the assembly experiment.

\section{Discussion}

In this work, we designed and fabricated a two-arm soft robot manipulator, and we carried out an experimental study on an assembly task under a binocular visual servo control system. Compared with the previous work, the presented soft robot uses dual soft robotic arms and incorporates binocular visual servo control.

In the previous work, a single-arm soft robot was designed, and the soft robots could not achieve the collaborative assembly task [7-9]. The dual-arm soft robot reported herein was able to realize collaborative work, however, which is critical for practical application.

Up to now, we have conducted several experiments on the fabricated dual-arm soft robot to verify its performance. As shown in Figure 10, the assembly task of the pipe joint and the air pipe was carried out to demonstrate that the collaborative work could be realized. In general, the process of achieving the cooperative work of the two arms is complicated, especially the design process of the visual servo control system based on the binocular camera, which involves image recognition, acquisition, processing, calculation of coordinate positions, and so on. In addition, it was implemented by the combination of software and hardware, such as solenoid valves, Arduino microcontrollers, high-definition cameras, etc. As compared to the previous works, the innovation of the presented work lies in the introduction of binocular cameras to achieve the cooperation of two soft manipulators. 
In addition, the camera was fixed at the base, and not the end of the manipulator arm. Furthermore, it has a very wide range of applications, such as in narrow workspaces, the assembly of tiny objects, and specific work demanding two-arm cooperation with high flexibility.

At the same time, the proposed soft robot has some shortcomings, such as insufficient precision in assembly, relatively low work efficiency, and limited weight of objects that can be grasped by the soft manipulator. In future work, further improvement will be performed in terms of the operation accuracy and work efficiency.

\section{Conclusions}

This paper presents the design, fabrication, and testing of a new dual-arm soft robotic manipulator for assembly tasks. Each arm consists of three soft modules and a soft end-effector, which are connected in series. One end-effector is a two-claw gripper and another is a four-claw gripper, which can cooperate with each other to execute more complex work than a single arm. The soft module is the basic element of the soft manipulator. The manufacturing process was described in detail. The evenly distributed cavities in each module were fabricated to produce the bending and elongation motion of the module. Specifically, the flexible bending of the module could be realized by changing the air pressure applied to the cavities. Silicone material was used to make the soft modules, which were highly flexible and could withstand a wide range of air pressure changes, making the module more flexible in space. Moreover, with the implemented visual servo control and air pressure control system, the soft manipulator was adopted to realize the assembly task of a pipe joint and a pipe. Experimental results demonstrate the effectiveness of the developed dual-arm soft robotic manipulator in cooperative assembly work. In the future work, more cooperative tasks will be carried out in experimental studies.

Author Contributions: Q.X. proposed the idea; Y.W. performed the literature view and experimental study; Y.W. and Q.X. wrote the paper.

Funding: This work was supported by the National Natural Science Foundation of China under Grant No. 51575545, and the Macao Science and Technology Development Fund under Grant No. 179/2017/A3.

Conflicts of Interest: The authors declare no conflict of interest. The founding sponsors had no role in the design of the study; in the collection, analyses, or interpretation of data; in the writing of the manuscript, and in the decision to publish the results.

\section{References}

1. Gerboni, G.; Ranzani, T.; Diodato, A. Modular soft mechatronic manipulator for minimally invasive surgery (MIS): Overall architecture and development of a fully integrated soft module. Meccanica 2015, 50, 2865-2878. [CrossRef]

2. De Falco, I.; Gerboni, G.; Cianchetti, M.; Menciassi, A. Design and Fabrication of an Elastomeric Unit for Soft Modular Robots in Minimally Invasive Surgery. J. Vis. Exp. 2015, 105, 53118. [CrossRef] [PubMed]

3. Yang, S.; Chen, W.; Liu, J.; Chen, W. Design, analysis and testing of a novel decoupled 2-DOF flexure-based micropositioning stage. J. Micromech. Microeng. 2017, 27, 095010. [CrossRef]

4. $\quad$ Dong, W.; Chen, F.; Gao, F.; Yang, M.; Sun, L.; Du, Z.; Tang, J.; Zhang, D. Development and analysis of a bridge-lever-type displacement amplifier based on hybrid flexure hinges. Precis. Eng. 2018, 54, 171-181. [CrossRef]

5. Xu, Q. Micromachines for Biological Micromanipulation; Springer: Cham, Switzerland, 2018.

6. Rus, D.; Tolley, M. Design, fabrication and control of soft robots. Nature 2015, 521, 467-475. [CrossRef] [PubMed]

7. Cianchetti, M.; Ranzani, T.; Gerboni, G.; De Falco, I.; Laschi, C.; Menciassi, A. STIFF-FLOP surgical manipulator: Mechanical design and experimental characterization of the single module. In Proceedings of the 2013 IEEE/RSJ International Conference on Intelligent Robots and Systems, Tokyo, Japan, 3-7 November 2013; pp. 3576-3581.

8. Harada, K.; Oetomoa, D.; Susilo, E.; Menciassia, A.; Daneya, D.; Merleta, J.-P.; Dario, P. A reconfigurable modular robotic endoluminal surgical system: Vision and preliminary results. Robotica 2010, 28, 171-183. [CrossRef] 
9. Steinemann, D.C.; Limani, P.; Clavien, P.A.; Breitenstein, S. Internal retraction in single-port laparoscopic cholecystectomy: Initial experience and learning curve. Minim. Invasive Therapy Allied Technol. 2013, 22, 171-176. [CrossRef] [PubMed]

10. Luo, M.; Tao, W.; Chen, F.; Khuu, T.K.; Ozel, S.; Onal, C.D. Design improvements and dynamic characterization on fluidic elastomer actuators for a soft robotic snake. In Proceedings of the 2014 IEEE International Conference on Technologies for Practical Robot. Applications (TePRA), Woburn, MA, USA, 14-15 April 2014; pp. 1-6.

11. Elsayed, Y.; Vincensi, A.; Lekakou, C.; Geng, T.; Saaj, C.M.; Ranzani, T.; Cianchetti, M.; Menciassi, A. Finite Element Analysis and Design Optimization of a Pneumatically Actuating Silicone Module for Robotic Surgery Applications. Soft Robot. 2014, 1, 255-262. [CrossRef]

12. Vikas, V.; Grover, P.; Trimmer, B. Model-free control framework for multi-limb soft robots. In Proceedings of the IEEE International Conference on Intelligent Robots and Systems, Hamburg, Germany, 28 September-3 October 2015; pp. 1111-1116.

13. Laschi, C.; Mazzolai, B.; Cianchetti, M.; Margheri, L.; Follador, M.; Dario, P. A soft robot arm inspired by the octopus. Adv. Robot. 2012, 26, 709-727. [CrossRef]

14. Fellmann, C.; Burgner-Kahrs, J. Implications of trajectory generation strategies for tubular continuum robots. In Proceedings of the IEEE International Conference on Intelligent Robots and Systems, Hamburg, Germany, 28 September-3 October 2015; pp. 202-208.

15. Yip, M.C.; Camarillo, D.B. Model-less feedback control of continuum manipulators in constrained environments. IEEE Trans. Robot. 2014, 30, 880-889. [CrossRef]

16. Zou, X.; Zou, H.; Lu, J. Virtual manipulator-based binocular stereo vision positioning system and errors modelling. Mach. Vis. Appl. 2012, 23, 43-63. [CrossRef]

17. Chen, Z.; Zhou, D.; Liao, H.; Zhang, X. Precision alignment of optical fibers based on telecentric stereo microvision. IEEE/ASME Trans. Mechatron. 2016, 21, 1924-1934. [CrossRef]

18. Suzumori, K.; Iikura, S.; Tanaka, H. Development of flexible micro-actuator and its applications to robotic mechanisms. In Proceedings of the 1991 IEEE International Conference on Robotics and Automation (ICRA), Sacramento, CA, USA, 9-11 April 1991; pp. 1622-1627.

19. Marchese, A.D.; Komorowski, K.; Onal, C.D.; Rus, D. Design and control of a soft and continuously deformable 2D robotic manipulation system. In Proceedings of the IEEE International Conference on Robotics and Automation, Hong Kong, China, 31 May-7 June 2014; pp. 2189-2196.

20. Arezzo, A.; Mintz, Y.; Allaix, M.E.; Arolfo, S.; Bonino, M.; Gerboni, G.; Brancadoro, M.; Cianchetti, M.; Menciassi, A.; Wurdemann, H.; et al. Total mesorectal excision using a soft and flexible robotic arm: A feasibility study in cadaver models. Surg. Endosc. 2017, 31, 264-273. [CrossRef] [PubMed]

21. Chang, B.; Chew, A.; Naghshineh, N.; Menon, C. A spatial bending fluidicactuator: Fabrication and quasistatic characteristics. Smart Mater. Struct. 2012, 21, 045008. [CrossRef]

22. Fischler, M.A.; Bolles, R.C. Random sample consensus: A paradigm for model fitting with applications to image analysis and automated cartography. Commun. ACM 1981, 24, 381-395. [CrossRef]

23. Niiyama, R.; Rus, D.; Kim, S. Pouch motors: Printable/inflatable soft actuators for robotics. In Proceedings of the 2014 IEEE International Conference on Robotics and Automation (ICRA), Hong Kong, China, 31 May-7 June 2014; pp. 6332-6337.

24. Polygerinos, P.; Wang, Z.; Overvelde, J.T.B.; Galloway, K.C.; Wood, R.J.; Bertoldi, K.; Walsh, C.J. Modeling of soft fiber-reinforced bending actuators. IEEE Trans. Robot. 2015, 31, 778-789. [CrossRef]

25. Wang, Z.; Hirai, S. A 3D printed soft gripper integrated with curvature sensor for studying soft grasping. In Proceedings of the 2016 IEEE/SICE International Symposium on System Integration, Sapporo, Japan, 13-15 December 2016; pp. 629-633.

26. De Falco, I.; Cianchetti, M.; Menciassi, A. A soft multi-module manipulator with variable stiffness for minimally invasive surgery. Bioinspir. Biomim. 2017, 12, 056008. [CrossRef] [PubMed]

(C) 2019 by the authors. Licensee MDPI, Basel, Switzerland. This article is an open access article distributed under the terms and conditions of the Creative Commons Attribution (CC BY) license (http:/ / creativecommons.org/licenses/by/4.0/). 